\title{
Mid-upper arm circumference: A surrogate for body mass index in pregnant women
}

\author{
A Fakier, ${ }^{1} \mathrm{MB}$ ChB, FCOG; G Petro, ${ }^{1,2} \mathrm{MB}$ ChB, FCOG; S Fawcus, ${ }^{3,4}$ MB ChB, FRCOG, MA \\ ${ }^{1}$ New Somerset Hospital, Cape Town, South Africa \\ ${ }^{2}$ Metro West, Cape Town, South Africa \\ ${ }^{3}$ Mowbray Maternity Hospital, Cape Town, South Africa \\ ${ }^{4}$ Department of Obstetrics and Gynaecology, Faculty of Health Sciences, University of Cape Town, South Africa
}

Corresponding author: A Fakier (ahminahf@gmail.com)

\begin{abstract}
Background. Nutrition in pregnancy has implications for both mother and fetus, hence the importance of an accurate assessment at the booking visit during antenatal care. The body mass index $\left(\mathrm{BMI}, \mathrm{kg} / \mathrm{m}^{2}\right)$ is currently the gold standard for measuring body fatness. However, pregnancy-associated weight gain and oedema, as well as late booking in our population setting, cause concern about the reliability of using the BMI to assess body fat or nutritional status in pregnancy. The mid-upper arm circumference (MUAC) has been used for many decades to assess malnutrition in children aged $<5$ years. Several studies have also shown a strong correlation between MUAC and BMI in both pregnant and non-pregnant adult populations.

Objective. To assess the correlation between the MUAC and BMI in pregnant women booking for antenatal care in the Metro West area of Cape Town, South Africa.

Methods. We conducted a cross-sectional study of women booking at four midwife obstetric units. Anthropometric measurements (height, weight and MUAC) were carried out on pregnant women at their first antenatal booking visit.

Results. The results showed a strong correlation between MUAC and BMI in pregnant women up to 30 weeks' gestation. The correlation was calculated at 0.92 for the entire group. The MUAC cut-offs for obesity (BMI $>30)$ and malnutrition (BMI <18.5) were calculated as $30.57 \mathrm{~cm}$ and $22.8 \mathrm{~cm}$, respectively.

Conclusion. MUAC correlates strongly with BMI in pregnancy up to a gestation of 30 weeks in women attending Metro West maternity services. In low-resource settings, the simpler MUAC measurement could reliably be substituted for BMI to assess nutritional status.
\end{abstract}

S Afr Med J 2017;107(7):606-610. DOI:10.7196/SAMJ.2017.vi7.12255

Nutrition in pregnancy has implications for both the mother and the fetus, hence the importance of an accurate assessment at the booking visit during antenatal care. Both over- and undernutrition can influence pregnancy outcomes. Obesity has major implications for both the mother and the fetus. ${ }^{[1]}$ The maternal risks include an increased risk for gestational diabetes and hypertension, caesarean section, venous thromboembolism, postpartum haemorrhage and sepsis. ${ }^{[1]}$ For the fetus, there is an increased risk of miscarriage, macrosomia, stillbirth and neural tube defects. ${ }^{[1]}$ Undernutrition is associated with anaemia, increased risks of infection, low birthweight and preterm delivery. ${ }^{[2]}$ Obesity is showing an alarming increase, and is currently more prevalent than undernutrition in the South African (SA) adult population. ${ }^{[3]}$ The fetal origins hypothesis was proposed by Barker $^{[4,5]}$ in the 1990s. According to this theory, fetuses exposed to poor nutrition in utero undergo adaptations that permanently change their physiology and metabolism. These programmed changes then predispose them to diseases in later life, specifically coronary heart disease, and the associated diseases of diabetes, hypertension and stroke. Whitaker ${ }^{[6]}$ looked at risk factors for childhood obesity and found that maternal obesity in early pregnancy doubles the risk of childhood obesity at age 2 - 4 years.

The body mass index $\left(\mathrm{BMI}, \mathrm{kg} / \mathrm{m}^{2}\right)$ is currently the gold standard for measuring body fatness. However, pregnancy-associated weight gain and oedema, as well as late booking into antenatal care in our population setting, causes us to question the reliability of using the BMI to assess body fat or nutritional status in pregnancy. Mid-upper arm circumference (MUAC) has been used for many decades to assess malnutrition in children aged $<5$ years. Several studies have shown a strong correlation between MUAC and BMI in adults. ${ }^{[7-12]}$

The MUAC is a much simpler anthropometric measure than the BMI, as its use eliminates the need for expensive equipment, such as height charts and scales, and the need for calculations. It is also much easier to perform on a patient who is acutely unwell, bed bound or sedentary. Another important advantage of using MUAC is that there is minimal change in the MUAC during pregnancy, so it may be a better indicator of pre-pregnancy body fat and nutrition than the BMI. ${ }^{[13]}$ It is measured midway between the olecranon of the elbow and the acromion process of the shoulder of the nondominant arm, using a standard tape measure. The use of the MUAC has been introduced as one of the routine anthropometric measurements at the antenatal booking visit in SA, and is recorded in the antenatal component of the standardised maternity case record as recommended by the current SA maternity care guidelines. ${ }^{[14]}$ These guidelines recommend the following: ${ }^{[14]}$

- MUAC $<23 \mathrm{~cm}$ (suggests malnutrition):

- Referral to dietician for nutritional support programme

- At risk of having a small-for-gestational-age baby.

- MUAC $>33 \mathrm{~cm}$ (suggests obesity):

- Screen for pre-eclampsia

- Screen for gestational diabetes

- Screen for macrosomia

- Requires a bigger blood pressure cuff. 


\section{Objective}

To assess the strength of the correlation between MUAC and BMI in pregnant women booking into antenatal care in the Metro West area of Cape Town, SA.

\section{Methods}

This was a cross-sectional study of women booking at four public sector midwife obstetric units (MOUs) in the Metro West area, Cape Town, SA, performed from 16 to 31 October 2014. The four MOUs, Retreat, Gugulethu, Hanover Park and Mitchell's Plain, all provide primary-level obstetric care and refer women with obstetric complications to specific referral hospitals in Metro West. They are usually the first entry point of women into the maternity system, and the majority of antenatal first visit bookings occur at these units. Although the individual social circumstances, living conditions and ethnicity of the participants were not captured for this study, it is known that all four MOUs serve women mainly of low socioeconomic status. They include both formal housing areas and informal settlements. All pregnant women booking at the study MOUs before 30 weeks' gestation were eligible for the study. Women beyond 30 weeks were excluded because the weight gain and oedema of the third trimester significantly influence BMI, whereas BMI in the earlier trimesters is more similar to pre-pregnancy BMI, which the MUAC reflects.

Exclusion criteria were: ( $i$ ) patients $<18$ years of age; (ii) gestational age $>30$ weeks at the booking visit; and (iii) twin gestation.

Anthropometric measurements of height, weight and MUAC were carried out on pregnant women booking for the first time at a gestation $<30$ weeks, in the four MOUs. The MUAC and weight measurements were done by the principal investigator using the same scale at each MOU. The height measurements were taken by the nurses at the various MOUs. The technique of measuring the MUAC was as described above.

The gestation was assessed by the best method available at each unit. An ultrasound machine was available at three of the units. At the Mitchell's Plain MOU, the symphysis-fundal height and dates were used to calculate the gestation.

\section{Statistical analysis}

The participants were divided into two groups, an early gestational age group (EGG) of patients who booked earlier than $\leq 20$ weeks + 0 days, and a late gestational age group (LGG) of those who booked at $\geq 20$ weeks +1 day but $\leq 29$ weeks +6 days.

The sample size was calculated as 164, based on the assumption that there would be a correlation between BMI and MUAC in $60 \%$ of patients $>20$ weeks' gestation (LGG), and $80 \%$ correlation in patients $<20$ weeks' gestation (EGG). A two-sided significance level of 95, power of $80 \%$ and ratio of $1: 1$ were used.

Pearson's correlation coefficient was used to calculate the correlation between BMI and MUAC. A linear regression analysis was performed to look at the mathematical relationship between BMI and MUAC and calculate the MUAC cut-offs for obesity and malnutrition.

\section{Ethical considerations}

Ethical approval for the study was obtained from the University of Cape Town Ethics Committee (ref. no. HREC \#644/2014) prior to data collection. Individual informed consent was obtained from all subjects.

\section{Results}

The required sample size of 164 women booking for antenatal care was obtained, and Table 1 provides limited demographic data on the study participants.
The distribution of BMI for the study subjects is shown in Table 2 and Fig. 1. The BMI ranged from 16.7 to $48.9 \mathrm{~kg} / \mathrm{m}^{2}$ with a mean (standard deviation (SD)) BMI calculated at $28.3(6.62) \mathrm{kg} / \mathrm{m}^{2}$. The $\mathrm{BMI}$ assessment revealed that $1.8 \%$ of participants were underweight, $32.9 \%$ fell into the normal BMI category, 32.3\% were overweight and $32.9 \%$ were obese.

The distribution of MUAC in the study subjects is shown in Table 3 and Fig. 2. The mean (SD) MUAC calculated was $29.4(4.83) \mathrm{cm}$ (range 20.6 - 44).

\begin{tabular}{ll}
\multicolumn{2}{l}{ Table 1. Demographic details of study subjects $(N=\mathbf{1 6 4})$} \\
\hline Participants from each MOU, $n$ \\
$\quad$ Gugulethu & 27 \\
Hanover Park & 10 \\
Retreat & 49 \\
Mitchell's Plain & 78 \\
Age (yr), mean (SD) & $27.09(5.56)$ \\
Age range (yr), $n(\%)$ & \\
18 - 23 & $52(31.7)$ \\
$24-29$ & $62(37.8)$ \\
30 - 35 & $36(21.9)$ \\
36 - 41 & $13(7.92)$ \\
42 - 47 & $1(0.6)$ \\
Parity, $n$ (\%) & \\
Primigravid & $46(28)$ \\
Multigravid & $118(71.9)$ \\
Weight (kg), mean (SD) & $72.5(17.7)$ \\
Height (cm), mean (SD) & $159.8(6.2)$ \\
MOU = midwife obstetric unit; SD = standard deviation. &
\end{tabular}

\begin{tabular}{ll}
\multicolumn{2}{l}{ Table 2. Distribution of BMI of participants $(\mathbf{N = 1 6 4 )}$} \\
\hline BMI category $\left(\mathbf{k g} / \mathbf{m}^{\mathbf{2}}\right)$ & $\boldsymbol{n}(\%)$ \\
\hline Underweight $(\leq 18.5)$ & $3(1.8)$ \\
Normal $(18.6-24.9)$ & $54(32.9)$ \\
Overweight $(25.0-29.9)$ & $53(32.3)$ \\
Obese $(30-39.9)$ & $45(27.4)$ \\
Morbidly obese $(\geq 40)$ & $9(5.5)$ \\
BMI = body mass index. &
\end{tabular}

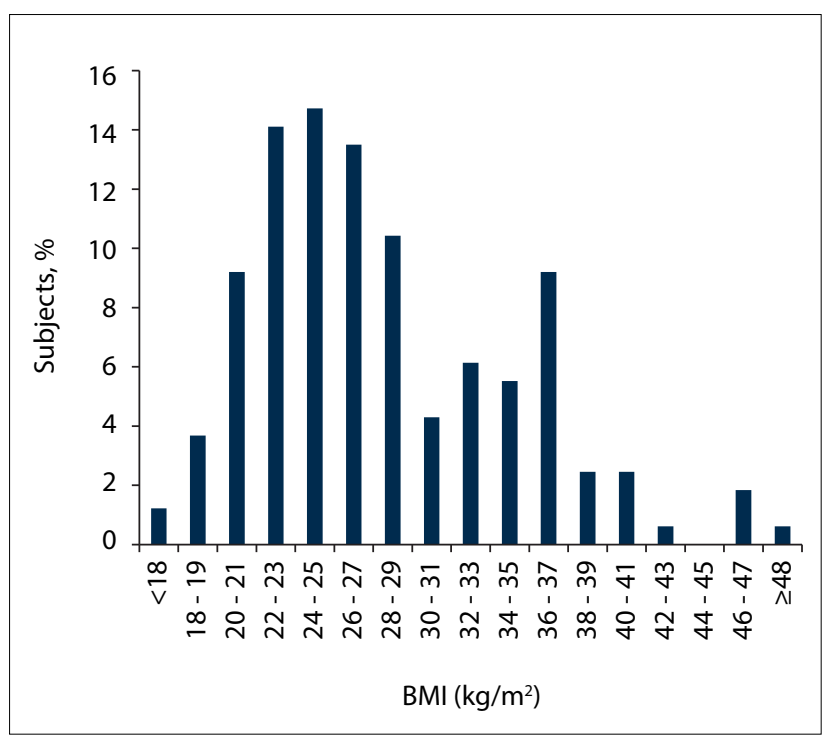

Fig. 1. Distribution of BMI. $(B M I=$ body mass index. $)$ 


\section{Table 3. Distribution of MUAC $(N=164)$}

\begin{tabular}{ll}
\hline MUAC range $(\mathbf{c m})$ & $\boldsymbol{n}(\%)$ \\
\hline $20-25$ & $33(20.1)$ \\
$25.1-30$ & $67(40.9)$ \\
$30.1-35$ & $38(23.2)$ \\
$35.1-40$ & $23(14.0)$ \\
$40.1-45$ & $3(1.8)$ \\
MUAC = mid-upper arm circumference. &
\end{tabular}

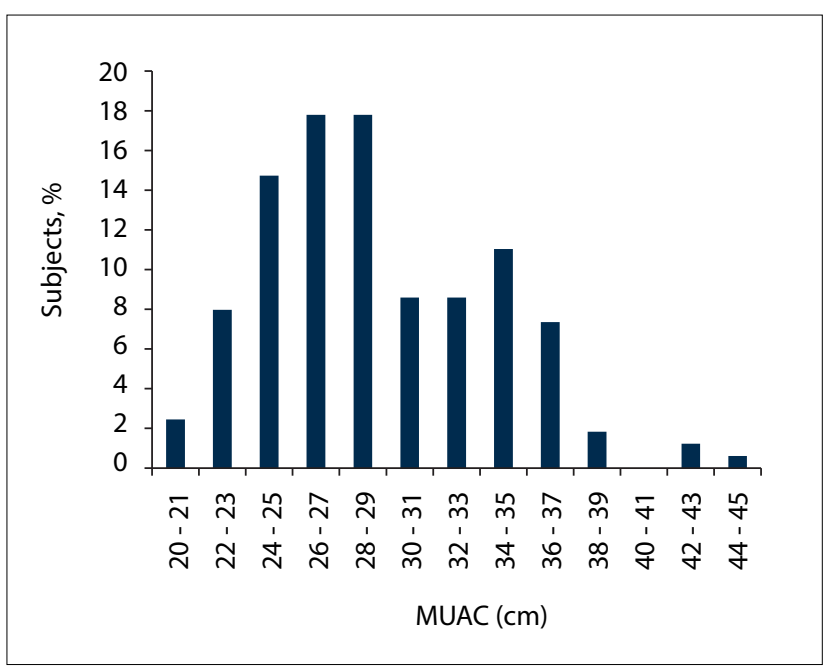

Fig. 2. Distribution of MUAC. (MUAC = mid-upper arm circumference. $)$

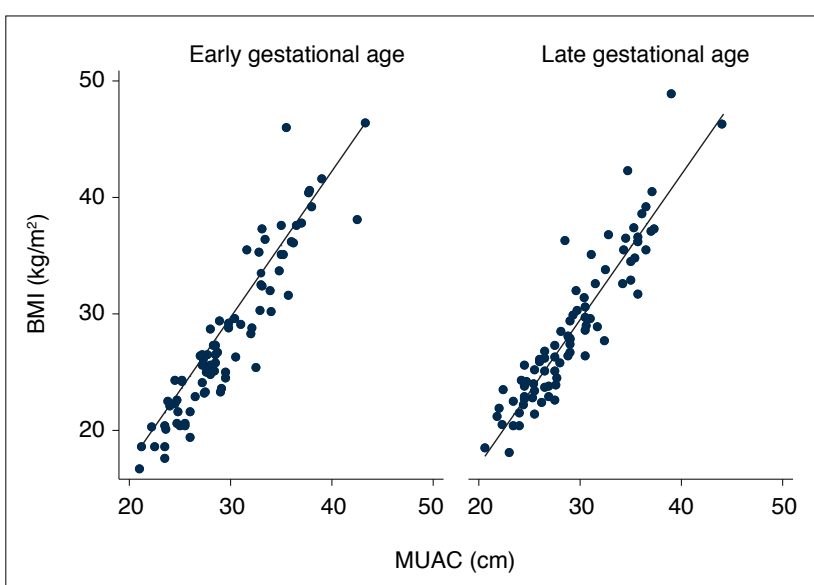

Fig. 3. Correlation between MUAC and BMI for the early and late gestational age groups. $(M U A C=$ mid-upper arm circumference; $B M I=$ body mass index.)

The correlation between BMI and MUAC for the EGG (gestation $<20$ weeks) was calculated as 0.93 and that for the LGG (gestation $>20$ weeks) as 0.92 . A visual presentation of this is shown in the scatter plot in Fig. 3.

The linear regression analysis showed that on average, for each $1 \mathrm{~cm}$ unit of MUAC, BMI increases by $1.27 \mathrm{~kg} / \mathrm{m}^{2}$ units. With a $p$-value of $<0.0001$, this represents a very strong statistical significance, i.e. the null hypothesis that there is no correlation is rejected and we could state that there was a statistically significant correlation between MUAC and BMI. This simple univariate model explained 0.85 $(85 \%)$ of the variation in BMI. To account for further variation, the possible role of gestational age in predicting the BMI was tested by the addition of gestational age as another variable, making it a multivariate analysis. The regression analysis showed that gestational age was also significant in predicting BMI $(p<0.0001)$. However, this effect could be different if we used only the two categories of $<20$ weeks (EGG) and $>20$ weeks (LGG). Once again the results were statistically significant although, as can be expected, at a weaker level $(p=0.002)$. From this regression analysis, the relationship between the three variables of BMI, MUAC and gestational age can be described mathematically:

In patients at $<20$ weeks' gestation:

BMI prediction $=-10.917+1.271 \times$ MUAC

In patients at $>20$ weeks' gestation:

BMI prediction $=-10.917+1.271 \times$ MUAC +1.2195

This can be further interpreted as follows: for every unit change in MUAC, BMI will increase by 1.27 units. Additionally, controlling for MUAC, LGG patients are predicted to have 1.2195 units of BMI more than EGG patients. This analytical method rests on the assumption that there is a linear relationship between the variables. This was tested by the visual inspection scatter plot displayed in Fig. 3. Furthermore, to use the results in the clinical setting, the cutoff points for different BMI categories were assessed.

Specificity, sensitivity, positive predictive value (PPV) and negative predictive value (NPV) were calculated for each MUAC cut-off (Table 4). Of note were the high specificity, sensitivity, PPV and NPV for the overweight and obese categories. The sensitivity and PPV were significantly lower in the morbidly obese category.

\section{Discussion}

Our study found a strong correlation of 0.92 between BMI and MUAC in pregnant women of $<30$ weeks' gestation in the Metro West maternity facilities studied. Surprisingly, this strong correlation persisted up to 30 weeks' gestation, disproving our hypothesis that the correlation would be weaker after 20 weeks' gestation because of the exponential increase in weight gain. The strong correlation is supported by a study by Cooley et al. ${ }^{[15]}$ on pregnant women in London and Ireland, which found a positive correlation of 0.836 between BMI and MUAC ( $p=0.01)$. Similarly, this strong correlation between BMI and MUAC was found by James et al. ${ }^{[7]}$ in five African countries in both men and women, as well as by Khadivzadeh ${ }^{[8]}$ in Iran, who studied 2000 non-pregnant women in the reproductive years.

A large proportion (71.9\%) of our participants were multiparous. Smith et al. ${ }^{[16]}$ found that during pregnancy, women gain body fat and experience a change in body fat distribution that persists after pregnancy. This altered body fat distribution may affect the MUAC of multiparous patients. However, this was not assessed in our study, and a suggestion for future studies would be to compare primigravid and multigravid patients.

MUAC cut-offs for the different BMI categories derived from the linear regression analysis are displayed in Table 5. We and Cooley et al.$^{[15]}$ had a MUAC cut-off point for overweight of $27 \mathrm{~cm}$. The MUAC cut-off for obesity in our study was 30.57 (95\% confidence interval (CI) 30.27 - 30.86) using a BMI of 30 as the gold standard for measuring obesity. This was rounded off to $31 \mathrm{~cm}$ for practical reasons. Of note, the Khadivzadeh ${ }^{[8]}$ study also had an obesity cut-off of $30.5 \mathrm{~cm}$ for BMI $>29 \mathrm{~kg} / \mathrm{m}^{2}$. Currently, the MUAC measurements used in the antenatal maternity case record in SA as normal values are $23-33 \mathrm{~cm}$. If $\mathrm{MUAC}$ is $>33 \mathrm{~cm}$, referral to a dietician for dietary advice is warranted, as well as screening for pre-eclampsia and gestational diabetes. The systematic review of Thangaratinam et al. ${ }^{[17]}$ showed that dietary intervention was the most successful intervention for 
Table 4. Specificity, sensitivity, NPV and PPV of MUAC for overweight, obese and morbidly obese BMI categories

\begin{tabular}{|c|c|c|c|}
\hline & $\begin{array}{l}\text { Overweight } \\
\left(\text { BMI } \geq 25 \mathrm{~kg} / \mathrm{m}^{2},\right. \\
\text { MUAC } \geq 27 \mathrm{~cm})\end{array}$ & $\begin{array}{l}\text { Obese } \\
\left(\text { BMI } \geq 30 \mathrm{~kg} / \mathrm{m}^{2},\right. \\
\text { MUAC } \geq 31 \mathrm{~cm})\end{array}$ & $\begin{array}{l}\text { Morbidly obese } \\
\left(B M I \geq 40 \mathrm{~kg} / \mathrm{m}^{2},\right. \\
\text { MUAC } \geq 37 \mathrm{~cm})\end{array}$ \\
\hline Sensitivity, \% (95\% CI) & $93.4(86.9-97.3)$ & $90.5(79.3-96.8)$ & $77.7(39.9-97.1)$ \\
\hline $\begin{array}{l}\mathrm{TP} /(\mathrm{TP}+\mathrm{FN}) \times 100 \\
(\text { screening test })\end{array}$ & $100 /(100+7) \times 100$ & $48 /(48+5) \times 100$ & $7(7+2) \times 100$ \\
\hline Specificity, \% (95\% CI) & $82.4(70.0-91.2)$ & $93.6(87.4-97.4)$ & $96.7(92.6-98.9)$ \\
\hline $\begin{array}{l}\mathrm{TN} /(\mathrm{TN}+\mathrm{FP}) \times 100 \\
(\text { diagnostic test })\end{array}$ & $47 /(10+47) \times 100$ & $104 /(7+104) \times 100$ & $150 /(5+150) \times 100$ \\
\hline PPV, \% (95\% CI) & $90.9(83.9-95.9)$ & $87.2(75.5-94.7)$ & $58.3(27.6-84.8)$ \\
\hline $\begin{array}{l}\mathrm{TP} /(\mathrm{TP}+\mathrm{FP}) \times 100 \\
\text { (likelihood of the condition) }\end{array}$ & $100 /(100+10) \times 100$ & $48 /(48+7) \times 100$ & $7 /(7+5) \times 100$ \\
\hline NPV, \% (95\% CI) & $87(75.0-94.6)$ & $95.4(89.6-98.4)$ & $98.6(95.3-99.8)$ \\
\hline $\begin{array}{l}\mathrm{TN} /(\mathrm{FN}+\mathrm{TN}) \times 100 \\
\text { (low probability of the condition) }\end{array}$ & $47 /(47+7) \times 100$ & $104 /(5+104) \times 100$ & $150 /(2+150) \times 100$ \\
\hline
\end{tabular}

Table 5. MUAC cut-off points for different BMI categories

\begin{tabular}{lll}
\hline BMI category & BMI $\left(\mathbf{k g} / \mathbf{m}^{2}\right)$ & $\begin{array}{l}\text { MUAC cut-off }(\mathbf{c m}) \\
\mathbf{( 9 5 \% ~ C I )}\end{array}$ \\
\hline Underweight & $\leq 18.5$ & $22.80(22.28-23.31)$ \\
Overweight & $25.0-29.9$ & $27.10(26.87-27.51)$ \\
Obese & $30.0-39.9$ & $30.57(30.27-30.86)$ \\
Morbidly obese & $\geq 40$ & $37.32(36.74-37.90)$
\end{tabular}

MUAC = mid-upper arm circumference; $\mathrm{BMI}=$ body mass index; $\mathrm{CI}=$ confidence interval.

reducing maternal weight gain and improving pregnancy outcomes, particularly in reducing the risk of pre-eclampsia. ${ }^{[17]}$ Screening for gestational diabetes is also advised, by performing a glucose tolerance test at 16 weeks and, if the results are normal, repeating it at 28 weeks. In addition, referral to a secondary-level institution for further antenatal care and delivery would be indicated. It is possible that the current MUAC cut-off for obesity of $>33 \mathrm{~cm}$ stipulated by the SA antenatal maternity case record is too high. According to our study the cut-off was calculated as $30.57 \mathrm{~cm}$, rounded off to $31 \mathrm{~cm}$ for practical reasons. Could this imply that we are missing a group of patients who are at risk and inadvertently assessing them as normal? Accepting these lower cut-offs would have definite financial implications as well as significantly increase the workload; to what benefit is unclear. Our study was not initially powered to calculate cut-offs, so a recommendation for further research would be to review and validate the current cut-off MUAC values.

The sensitivity and PPV of MUAC drops significantly in the morbidly obese group of patients, at $77.7 \%$ (95\% CI 39.9 - 97.1) and $58.3 \%$ (95\% CI 27.6 - 84.8), respectively (Table 5). For this category, using BMI instead of MUAC is recommended by the SA maternity care guidelines.

Okereke et al ${ }^{[9]}$ studied anthropometric indices for diagnosing obesity in pregnancy in a cross-sectional study in Nigeria. They found that MUAC of $33 \mathrm{~cm}$ and a calf circumference of $39 \mathrm{~cm}$ may be a reliable cut-off for diagnosing obesity in pregnant women in Nigeria. Interestingly, in the Okereke study, MUAC had a strong positive correlation with maternal weight (BMI), but there was no significant difference in the three trimester groups, suggesting that MUAC is independent of gestational age and could be used to identify obesity in women regardless of the duration of pregnancy. This disputes our concern that weight gain in the third trimester would significantly impact on the correlation of MUAC with BMI. However, there have been different MUAC cut-offs internationally for undernutrition (MUAC $<18.5 \mathrm{~cm}$ (Zimbabwe 2008), $<21.0 \mathrm{~cm}$ (Burkina Faso, Burundi 2002, DRC 2008, Guinea 2005, Madagascar 2007, Malawi 2007, Mali 2007, Nigeria 2006, Senegal 2008), $<22.0 \mathrm{~cm}$ (Mozambique 2008), $<22.5 \mathrm{~cm}$ (Zambia 2009), $<23.0 \mathrm{~cm}$ (Indonesia 1996) and $\leq 23 \mathrm{~cm}$ (Sri Lanka 2006)), ${ }^{[18]}$ and there are probably different cut-offs for measuring obesity as well.

Our cut-off for malnutrition was measured at $22.8 \mathrm{~cm}$ (rounded off to $23 \mathrm{~cm}$ ), which is consistent with the current MUAC values used in the maternity care guidelines for referral to a dietician. Despite the malnutrition group being quite small, our cut-offs were consistent with those found by Sultana et al., ${ }^{[10]}$ who looked at MUAC cut-off for undernutrition in Bangladeshi adults. Their findings provided MUAC measurements of $<24 \mathrm{~cm}$ and $<25 \mathrm{~cm}$ for females and males respectively for detecting undernutrition in adults. The underweight group of women comprised a mere $1.8 \%$ of subjects, which is consistent with Puoane et al., ${ }^{[3]}$ who found that underweight women were rare in SA in an obesity health survey study in 2002, where underweight women represented only $5.6 \%$ of their subjects. Although the provision of nutritional support to malnourished women remains controversial, it would be difficult to ignore the opportunity of pregnancy to provide access into a nutritional programme to improve their health, and potentially reduce adverse pregnancy outcomes.

The BMI distribution of the population of pregnant women sampled is in agreement with the study by Puoane et al. ${ }^{[3]}$ mentioned above, in which $56.6 \%$ of females aged $\geq 15$ years were found to be overweight or obese. Our percentage for overweight and obesity in this group is an alarming $65.1 \%$, representative of increasing concern over the obesity epidemic. Fig. 1 displays the BMI distribution. A bimodal distribution in the obese category is evident from the bar chart. Our study did not follow up participants to assess outcomes, but to do so would be of interest for future studies. A retrospective cross-sectional study by Basu et al. ${ }^{[19]}$ on obesity and its outcomes in pregnant women in SA looked at 767 women and found that $44 \%$ were classified as obese or morbidly obese according to World Health Organization BMI classification. They also found that urinary tract infection, failed induction of labour and gestational diabetes were more common in the morbidly obese category. The percentage of women found to be obese or morbidly obese in our study was not as high as in the Basu study. However, an alarming $32.8 \%$ fell 
into these categories. The prevalence of obesity is rising globally. It is a risk factor for non-communicable diseases such as ischaemic heart disease, type 2 diabetes, hypertension and cancer. ${ }^{[19]} \mathrm{SA}$ faces a double burden of disease, where undernutrition (mainly in children) and obesity may coexist in the same household, family and community. ${ }^{[20]}$ Urbanisation of South Africans has certainly contributed to the increase in obesity, with a change from a traditional low-fat, high-fibre diet to a more Western diet containing high fat and more refined foods. ${ }^{[21]}$ In certain communities obesity is often seen culturally as a sign of health, affluence and respect, whereas thinness is associated with illness and HIV. ${ }^{[22]}$ This contributes to a mindset of 'big is beautiful', resulting in few women who are obese actually perceiving themselves as such. ${ }^{[22]}$

\section{Study limitations}

There were some study limitations. The lack of a standardised method for calculating the exact gestational age was definitely a limitation, along with the lack of demographic, medical and social data. A strength of the study was that a single provider took the measurements, enabling consistency for testing the correlation of BMI and MUAC. However, this could also be perceived as a limitation in that an assessment of reproducibility of the MUAC measurements was not done.

The mean gestation was 19 weeks and the cut-off of 30 weeks' gestation was quite limiting. In retrospect, when comparing patients who booked at $<20$ weeks with those at $>20$ weeks, it may have been advisable to have included patients up to 40 weeks' gestation, as the majority of weight gain occurs in the third trimester. Further research needs to focus on the use of MUAC between 30 and 40 weeks' gestation.

\section{Conclusion}

The MUAC correlates strongly with BMI in pregnancy at the booking visit, up to a gestation of 30 weeks in women attending Metro West maternity services.

In low-resource settings, the simpler MUAC measurement to assess nutritional status and screen women who are at risk for potential adverse pregnancy outcomes could reliably be substituted for BMI estimation. This would eliminate the need for calibrated weight scales and height charts, as well as calculations for BMI.

Acknowledgements. Elizabeth Botha is acknowledged for performing the statistical analysis and Prof. Sue Fawcus and Dr Greg Petro for their supervision of the study.

Author contributions. AF: research design, development of the protocol, data collection and preparation of the article; SF: provided the research topic, supervision of the study and article development; GP: provided assistance in research design, sample size calculation and article development.

Funding. None.

Conflicts of interest. None.

1. Dixit A, Girling JC. Obesity and pregnancy. J Obstet Gynaecol 2008;28(1):14-23. https://dx.doi org $/ 10.1080 / 01443610701814203$

2. Cox JT, Phelan ST. Nutrition during pregnancy. Obstet Gynecol Clin North Am 2008;35(3):369-383. https://dx.doi.org/10.1016/j.ogc.2008.04.001

3. Puoane T, Steyn K, Bradshaw D, et al. Obesity in South Africa: The South African Demographic and Health Survey. Obes Res 2002;10(10):1038-1048. https://dx.doi.org/10.1038/oby.2002.141

4. Barker JP. Maternal nutrition, fetal nutrition, and disease in later life. Nutrition 1997;13(9):807-813. Barker JP. Maternal nutrition, fetal nutrition,
https://doi.org/10.1016/s0899-9007(97)00193-7

5. Barker JP. The fetal and infant origins of adult disease. BMJ 1990;301(6761):1111. https://doi Barker JP. The fetal and infant
org/10.1136/bmj.301.6761.1111

org $/ 10.1136 / \mathrm{bmj} .301 .6761 .1111$
6. Whitaker R. Predicting preschooler obesity at birth: The role of maternal obesity in early pregnancy. Whitaker R. Predicting preschooler obesity at birth: The role of mater
Pediatrics 2004;114(1):e29-e36. https://doi.org/10.1542/peds.114.1.e29

7. James WP, Mascie-Taylor GC, Norgan NG, Bistrian BR, Shetty PS, Ferro-Luzzi A. The value of arm circumference measurements in assessing chronic energy deficiency in third world adults. Eur J Clin Nutr 1994;48(12):883-894.

8. Khadivzadeh T. Mid upper arm and calf circumferences as indicators of nutritional status in women of reproductive age. East Mediterr Health J 2002;8(4-5):612-618

9. Okereke C, Anyaehie U, Dim C, Iyare E, Nwagha U. Evaluation of some anthropometric indices for the diagnosis of obesity in pregnancy in Nigeria: A cross-sectional study. Afr Health Sci 2013;13(4):10341040. https://dx.doi.org/10.4314\%2Fahs.v13i4.25

10. Sultana T, Karim MN, Ahmed T, Hossain MI. Assessment of under nutrition of Bangladeshi adults using anthropometry: Can body mass index be replaced by mid-upper-arm-circumference? PLoS One 2015;10(4):e0121456 https://dx.doi.org/10.1371/journal.pone. 0121456

11. Nguyen P, Ramakrishnan U, Katz B, et al. Mid-upper-arm and calf circumferences are useful predictors Nguyen P, Ramakrishnan U, Katz B, et al. Mid-upper-arm and calf circumferences are useful predictors
of underweight in women of reproductive age in northern Vietnam. Food Nutr Bull 2014;35(3):301of underweight in women of reproductive age in north

12. Chakraborty R, Bose $K$, Koziel $S$. Use of mid-upper arm circumference in determining undernutrition and illness in rural adult Oraon men of Gumla District, Jharkhand, India. Rural Remote Health 2011;11(3):1754.

13. Lopez LB, Calvo E, Poy M, del Valle Balmaceda Y, Camera K. Changes in skinfolds and mid-upper arm circumference during pregnancy in Argentine women. Matern Child Nutr 2011;7(3):253-262. https:/ doi.org/10.1111/j.1740-8709.2009.00237.x

4. National Department of Health, South Africa. Guidelines for Maternity Care in South Africa: A Manual for Clinics, Community Health Centres and District Hospitals. 4th ed. Pretoria: NDoH, 2014.

15. Cooley SM, Donnelly J, Walsh T, et al. The relationship between body mass index and mid-arm circumference in a pregnant population. J Obstet Gynaecol 2011:31(7):594-596. https://dx.doi.org/ 10.3109/01443615.2011.597892

16. Smith DE, Lewis CE, Caveny JL, Perkins LL, Burke GL, Bild DE. Longitudinal changes in adiposity associated with pregnancy: The Cardia Study: Coronary Artery Risk Development In Young Adults associated with pregnancy: The Cardia Study: Coronary Artery Risk Development In Young
Study. JAMA 1994;271(22):1747-1751. https://dx.doi.org/10.1001/jama.1994.03510460039030

17. Thangaratinam S, Rogozinska E, Jolly $K$, et al. The effects of interventions in pregnancy on maternal weight and obstetric outcomes: Meta-analysis of randomised evidence. BMJ 2012;344:e2088. https:// dx.doi.org/10.1136/bmj.e2088

18. Ververs M, Antierens A, Sackl A, Staderini N, Captier V. Which anthropometric indicators identify a pregnant woman as acutely malnourished and predict adverse birth outcomes in a humanitarian context? PLoS Curr 2013;5. https://dx.doi.org/10.1371/currents.dis.54a8b618c1bc031ea140e3f2934599c8

19. Basu J, Jeketera C, Basu D. Obesity and its outcomes among pregnant South African women. Int J Gynaecol Obstet 2010;110(2):101-104. https://dx.doi.org/10.1016/j.ijgo.2010.02.020

20. Vorster HH. The link between poverty and malnutrition: A South African perspective. Health SA Gesondheid 2009;5(1):435. https://dx.doi.org/10.4102/hsag.v15i1.435

21. Vorster H, Venter C, Wissing M, Margets B. The nutrition and health transition in the North West Province of South Africa: A review of the THUSA (Transition and Health during Urbanisation of
South Africans) study Public Health Nutr 2005:8(5):480-490. https://dx.doi.org/10.1079/PHN200578

22. Kouth Africans) study. Public Health Nutr 2005;8(5):480-490. https.//dx.doi.org/10.1079/PHN200578 Kruger HS, Puoane T, Senekal M, van der Merwe M-T. Obesity in South Africa: Challenges
for government and health professionals. Public Health Nutr 2005;8(5):491-500. http://dx.doi. for government and hea
org/10.1079/PHN2005785

Accepted 21 February 2017. 